\title{
Improvement of scanning probe microscopy local oxidation nanolithography
}

\author{
Shinya Nishimura, Takashi Toyofuku, and Kazuya Miyashita \\ Department of Electrical and Electronic Engineering, Tokyo University of Agriculture and Technology, \\ Koganei, Tokyo 184-8588, Japan \\ Yasushi Takemura \\ Division of Electrical and Computer Engineering, Yokohama National University, Hodogaya, \\ Kanagawa 240-8501, Japan \\ Jun-ichi Shirakashi ${ }^{\text {a) }}$ \\ Department of Electrical and Electronic Engineering, Tokyo University of Agriculture and Technology, \\ Koganei, Tokyo 184-8588, Japan
}

(Received 20 August 2008; accepted 9 February 2009; published 1 April 2009)

\begin{abstract}
In order to investigate the factors that control the growth of Si local oxide, the authors have a comparative study between tapping and contact mode scanning probe microscopy (SPM) local oxidation experiments using the same SPM tip. The authors fabricated Si oxide wires with an average full width at half maximum (FWHM) of $11.0 \mathrm{~nm}$ in tapping mode and $54.8 \mathrm{~nm}$ in contact mode under the same oxidation conditions. The standard deviations of the FWHM were 2.1 and 12.2 $\mathrm{nm}$ in tapping and contact mode experiments, respectively. Furthermore, the authors quantitatively explained the size of the oxide controlled with tapping mode local oxidation using a model based on the oxidation ratio and the rate constant of the oxidation reaction. The oxidation ratio was estimated to be approximately $10 \%$ in tapping mode oxidation, which contributes to the $10 \mathrm{~nm}$ scale resolution of the oxide. Moreover, the rate constants in the tapping mode local oxidation reaction were larger than those of the contact mode oxidation. This indicates that local oxidation reaction controlled with tapping mode operation is enhanced and results in the stable oxidation, since space charges accumulated in the oxide are easily neutralized by the modulation of the electric field strength. The results imply the use of tapping mode operation for performing controllable local oxidation nanolithography. (C) 2009 American Vacuum Society. [DOI: 10.1116/1.3093907]
\end{abstract}

\section{INTRODUCTION}

Local oxidation nanolithography using scanning probe microscopy (SPM) has been widely used to produce nanometer-scale structures on the substrate surface. ${ }^{1}$ This technique is recognized as possible nanofabrication tools for nanoelectronic applications such as single-electron transistors ${ }^{2,3}$ and planar-type ferromagnetic tunnel junctions. ${ }^{4,5}$ In general, the feature size of the oxide is strongly affected by the size of the water meniscus, ${ }^{6}$ relative humidity, ${ }^{7}$ applied bias voltage, ${ }^{8}$ scanning speed of the SPM cantilever, ${ }^{8}$ and operation mode. ${ }^{9}$ It is also known that the voltage modulation in contact mode SPM local oxidation overcomes the self-limiting property of the oxidation by eliminating the buildup of space charges within the oxide during growth. ${ }^{10}$ Direct comparison between contact and tapping mode SPM local oxidation experiments has been provided on the size uniformity of $\mathrm{Ni}$ and $\mathrm{Fe}$ oxide wires, and found that the tapping mode SPM local oxidation produces stable oxidation. ${ }^{11}$ Recently, notable improvements in the size controllability and uniformity have been studied through the control of dynamic properties of cantilever. ${ }^{12}$ Moreover, we have achieved the sub-10-nm resolution of tapping mode SPM local oxidation nanolithography with optimization of cantilever dynamics. ${ }^{13}$ It is considered that tapping mode SPM local oxidation is a suitable technique for producing

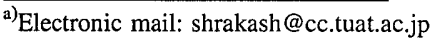

oxide wires with good size controllability and uniformity. However, different cantilevers are usually used in comparative studies between contact and tapping mode SPM local oxidation experiments. Ideally, the same cantilever should be used in both local oxidation methods since the size of oxide depends on the tip's size and geometry.

For local oxidation kinetics, there has been a quantitative interpretation of experimental kinetic data for SPM oxidation. ${ }^{14}$ In contact mode oxidation, an analytical model that explains the behavior of the oxide height with the applied bias voltage and the scanning speed was proposed. ${ }^{15}$ It was also reported that the growth rate dependence of Si oxide on the oxidation time is investigated with the rate constant for contact mode oxidation. ${ }^{16}$ Furthermore, we showed a simple model of tapping mode SPM local oxidation based on the oxidation ratio, which is defined as the oxidation time divided by the period of the cantilever oscillation. ${ }^{13}$

In this article, in order to clear the mechanism of SPM local oxidation, we have a comparative study of contact and tapping mode SPM local oxidation experiments with the same SPM tip. In addition, we estimate the oxidation ratio and rate constant for the fabrication of $\mathrm{Si}$ oxide wires with 10 $\mathrm{nm}$ resolution.

\section{EXPERIMENTAL METHODS}

The experiments were performed using a SPM unit (SPA400/SPI4000, SII Nano'Technology) at room tempera- 
ture. The dc bias voltage was applied to $p$-type silicon (100) with a resistivity of $1 \mathrm{k} \Omega \mathrm{cm}$. The silicon native oxide was removed by dipping the sample in HF solution. Ambient air humidity was maintained at $20 \%$. In order to perform a direct comparison between contact and tapping mode experiments, we have kept unchanged the sample properties, the relative humidity, and other control parameters of $\mathrm{Si}$ oxide wires such as bias voltage and scanning speed. Furthermore, the same SPM cantilever was used between both methods. The spring constant and resonance frequency of the Si cantilever were $40 \mathrm{~N} / \mathrm{m}$ and $300 \mathrm{kHz}$, respectively. The tip radius is 7 $\mathrm{nm}$. In the experiments, the local oxidation in contact mode operation was performed by applying a contact force as low as possible, which is $78 \mathrm{nN}$, in order to avoid wear of SPM tip. Because the tip is in contact with the surface, the stiff cantilever is not generally suitable for the contact mode operation. In contact mode SPM local oxidation, we confirmed that the cantilever is approached to the sample surface during the oxidation by monitoring the deflection of the cantilever. With this method, it is possible to perform contact mode SPM local oxidation with stiff cantilever at lower contact force. In tapping mode SPM local oxidation, the oscillation amplitude of the cantilever was set at $292 \mathrm{~nm}$ by tuning the excitation voltage of the cantilever.

\section{RESULTS AND DISCUSSION}

Figure 1 shows SPM images of (a) contact and (b) tapping mode SPM local oxidation experiments using the same SPM tip at different dc voltages and scanning speeds. The applied bias voltage and scanning speed were varied from 17.5 to $22.5 \mathrm{~V}$ and from 250 to $750 \mathrm{~nm} / \mathrm{s}$, respectively. All SPM images in this study were of areas of $500 \times 500 \mathrm{~nm}^{2}$. A visual inspection reveals that the Si oxide wires of the tapping mode oxidation are smaller than those of contact mode oxidation. Moreover, the size fluctuation of the Si oxide wires is further suppressed in tapping mode experiments. The cross sections of the Si oxide wires were taken along the $X$ axis in Figs. 1(a) and 1(b). Figures 2(a) and 2(b) show the crosssectional profiles of the Si oxide wire in contact and tapping mode oxidation with the applied voltage of $22.5 \mathrm{~V}$ and the scanning speed of $750 \mathrm{~nm} / \mathrm{s}$. Average full width at half maximum (FWHM), total width (TW), and height were calculated from cross-sectional profiles. To evaluate the size uniformity of the Si oxide wires, the standard deviations (STDs) of FWHM, TW, and height were also determined from the cross sections. From Fig. 2(b), since the STD of FHWM in tapping mode oxidation is $2.1 \mathrm{~nm}$, the size fluctuation of the oxide is further suppressed than that of contact mode experiments shown in Fig. 2(a) (STD of FWHM=12.2 nm). In Fig. 3(a), the FWHM and TW of the Si oxide wires are plotted as a function of scanning speed (dc voltage $=22.5 \mathrm{~V}$ ). Furthermore, the height and its STD are shown in Fig. 3(b). As widely known in SPM local oxidation, the size of the oxide is controlled by changing the scanning speed in both contact and tapping mode oxidation experiments. By changing the scanning speed from 250 to $750 \mathrm{~nm}$, the feature size of the oxide in contact mode oxidation is ranged from 62.7 to 54.8
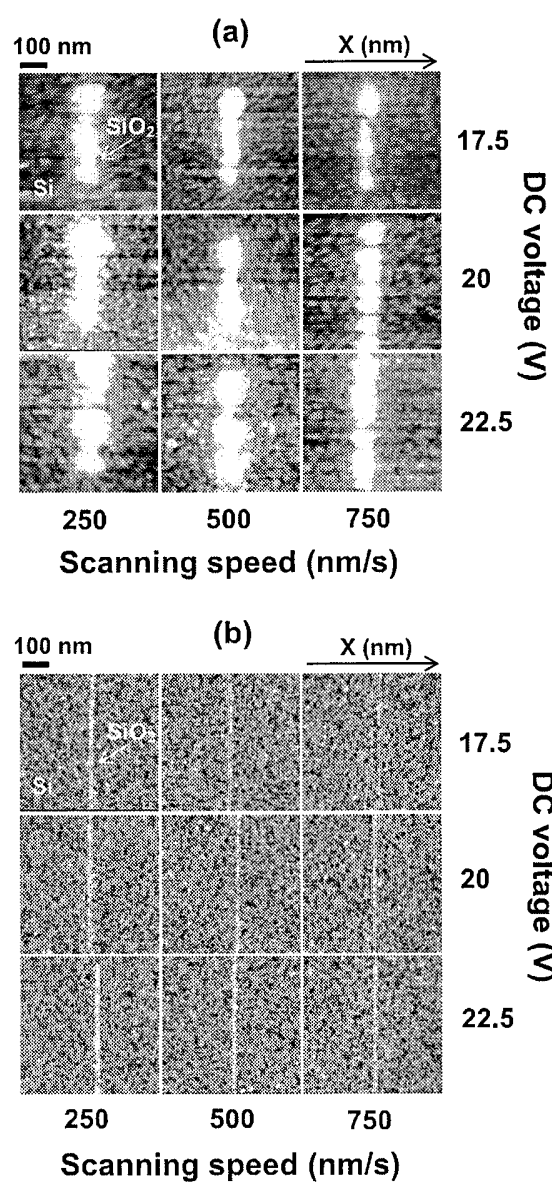

FIG. 1. (Color online) SPM images of (a) contact mode and (b) tapping mode SPM local oxidation experiments with different dc bias voltages and scanning speeds. The same SPM tip is used between contact and tapping mode local oxidation.

$\mathrm{nm}$ in FWHM and from 2.8 to $2.5 \mathrm{~nm}$ in height, respectively. Additionally, with increasing the scanning speed, the FWHM and height of the oxide in tapping mode oxidation also decreased from 16.3 to $11.0 \mathrm{~nm}$ and from 0.6 to $0.5 \mathrm{~nm}$, respectively. This result indicates that the smaller resolution of the oxide is realized by tapping mode operation. Furthermore, Fig. 3(c) shows the STDs of FWHM and TW of the Si oxide wires. All STDs of FWHM in tapping mode oxidation are below $5 \mathrm{~nm}$, which are smaller than those obtained by contact mode experiments. The relative STDs (RSDs) of FWHM of Si oxide wires fabricated by tapping mode oxidation are approximately $20 \%$. A similar behavior is also seen in tapping mode $\mathrm{Si}$ oxide wires with RSDs higher than $10 \%{ }^{17}$ Since local oxidation experiments are performed under the same conditions including the cantilever, the feature size of the Si oxide wires is strongly affected by SPM modes of operation.

In order to understand the advantages of tapping mode operation on both controllability and uniformity of the oxide formation, the oxidation reaction induced by the tapping mode operation is considered with a model based on the 

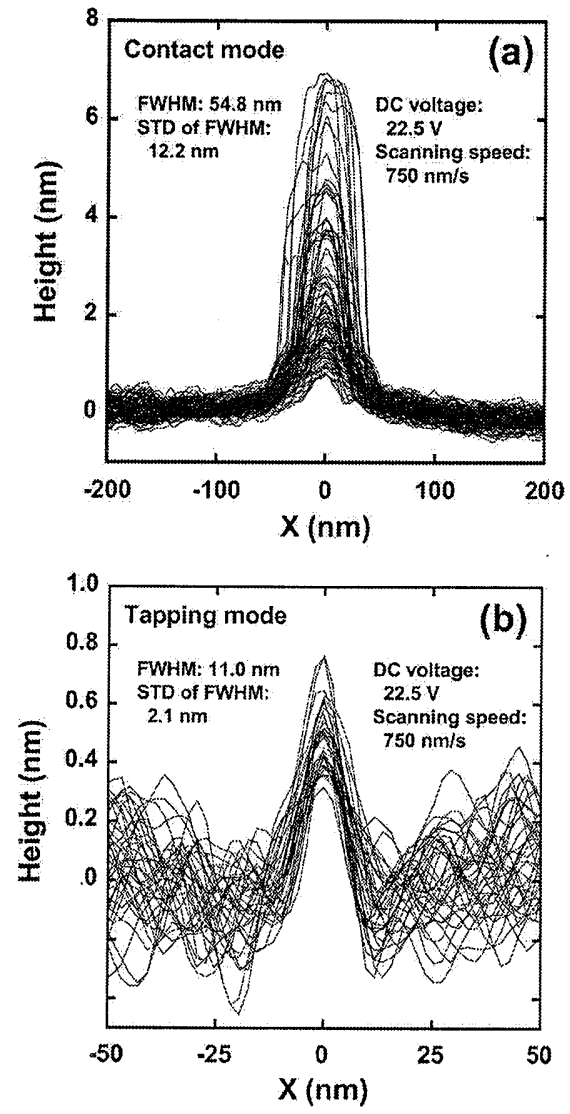

FIG. 2. Cross sections of Si oxide wires fabricated by (a) contact mode and (b) tapping mode SPM local oxidation experiments using the same SPM tip (dc bias voltage $=22.5 \mathrm{~V}$, scanning speed $=750 \mathrm{~nm} / \mathrm{s}$ ). The cross sections were taken along the $X$ axis.

oxidation ratio and the rate constant. An analytical model of tapping mode SPM local oxidation with respect to the oxidation ratio $R$ is expressed as follows: ${ }^{13}$

$$
R(\%)=\frac{\tau}{T}=\left(\frac{1}{\pi} \sin ^{-1}\left(\frac{2 D-A}{A}\right)+0.5\right) \times 100,
$$

where $\tau$ is the oxidation time, $T$ is the period of cantilever oscillation, $A$ is the oscillation amplitude of the cantilever, and $D$ is the maximum length of stretched water meniscus. Figure 4 shows the oxidation ratio and the FWHM of Si oxide wires as function of the oscillation amplitude of the cantilever. Solid circles represent the experimental data with the scanning speed of $20 \mathrm{~nm} / \mathrm{s}$. With enhancing the oscillation amplitude of the cantilever from 23 to $432 \mathrm{~nm}$, the FWHM of the oxide is clearly decreased from 67 to $20 \mathrm{~nm}$. This analytical model provides a good fit to the experimental data when the $D$ ranges from 10 to $25 \mathrm{~nm}$. It is considered that contact mode oxidation has no dwell time and shows the $100 \%$ oxidation ratio. In this fitting model, the $100 \%$ oxidation ratio corresponds to the $150 \mathrm{~nm}$ oxide width, which is consistent with the maximum width of the $\mathrm{Si}$ oxide formed by the conventional contact mode oxidation. ${ }^{9}$ Furthermore, the experimental data $(V=22.5 \mathrm{~V})$ of tapping mode oxida-
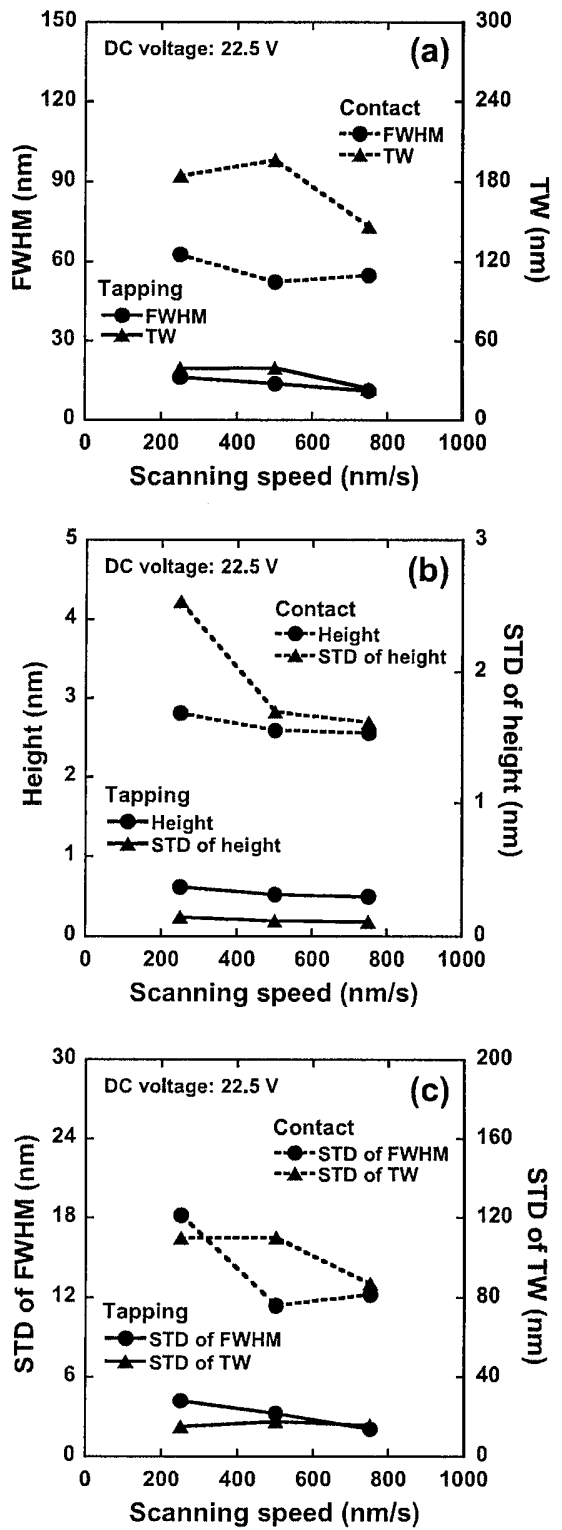

FlG. 3. Dependence of (a) FWHM and TW, (b) height and its standard deviation, and (c) standard deviations of FWHM and TW of Si oxide wires on scanning speed of the cantilever.

tion in Fig. 1(b) are also plotted in Fig. 4. Open triangle, square, and circle represent the FWHM of the oxide when the scanning speed is set at 250,500 , and $750 \mathrm{~nm} / \mathrm{s}$, respectively. As shown in this figure, maximum length of the water meniscus is deduced to be approximately $10 \mathrm{~nm}$ in our fitting procedure. Hence, it is suggested that the oxidation ratio becomes $10 \%-16 \%$ in order to realize the stable local oxidation with sub-10-nm resolution. Since the anodic oxidation occurs within the water meniscus, the average lateral dimension of the water meniscus during the tapping mode oxidation is around $10 \mathrm{~nm}$ in this situation. Additionally, the average intensity of electric field strength is reduced due to 


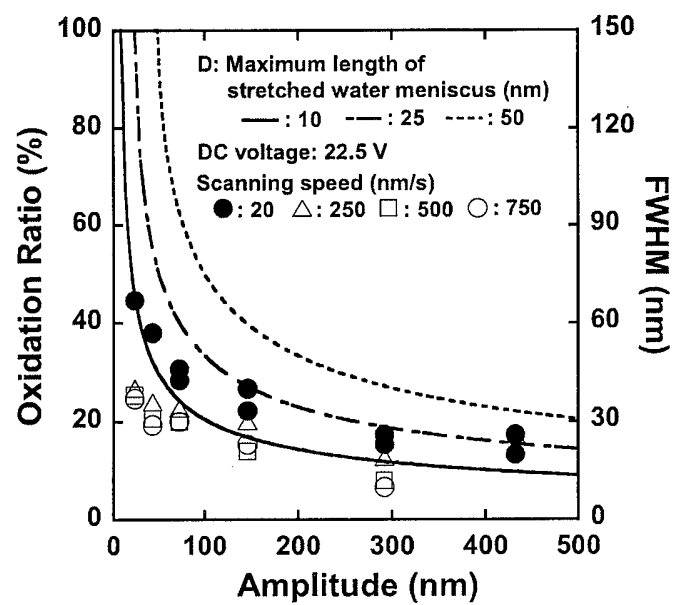

FIG. 4. Oxidation ratio and FWHM as function of oscillation amplitude. dc bias voltage is set at $22.5 \mathrm{~V}$. Solid circles represent the experimental data with the scanning speed of $20 \mathrm{~nm} / \mathrm{s}$ as the oscillation amplitude of cantilever is varied from 23 to $432 \mathrm{~nm}$. Open triangle, square, and circle represent the FWHM of the oxide as the scanning speed is set at 250,500 , and $750 \mathrm{~nm} / \mathrm{s}$, respectively.

tapping mode operation, so that this also contributes to the smaller resolution of the oxide. ${ }^{18}$

Here, we consider the rate constant $k \mathrm{~s}^{-1}$ for SPM local oxidation reaction. In general, the rate constant is determined by measuring the concentration of the species for the reaction. ${ }^{19}$ In accordance with the model described by $\mathrm{Da}-$ gata et al. ${ }^{16}$ the height of the Si oxide is proportional to the concentration of $\mathrm{SiO}_{2}$ and is expressed in the following formula:

$$
\begin{aligned}
h(t) & \propto\left[\mathrm{SiO}_{2}(t)\right] \\
& =\left[A_{0}\right]\left(1-e^{-k_{3} t}+\frac{k_{4}-k_{3}}{k_{3}-\left(k_{1}+k_{4}\right)}\left(e^{-\left(k_{1}+k_{4}\right) t}-e^{\left.-k_{3} t\right)}\right),\right.
\end{aligned}
$$

where $h(t)$ is the oxide height, $\left[\mathrm{SiO}_{2}(t)\right]$ is the evolution of the $\mathrm{SiO}_{2}$ concentration, $t$ is the oxidation duration, $\left[A_{0}\right]$ is the initial concentration of oxyanions, $k_{4}$ is the rate constant for the direct oxidation process, and $k_{1}$ and $k_{3}$ are the rate constants for indirect reaction mediated by trapped charge defects at the growing interface. In this model, Dagata also shows that the empirical power low, $\left[\mathrm{SiO}_{2}\left(t^{\gamma}\right)\right]$, provides a good fit to the experimental data, where $\gamma$ is 0.4 for contact mode SPM local oxidation. We investigate the rate constant between contact and tapping mode SPM local oxidation experiments. Oxidation was performed under the applied bias voltage of $22.5 \mathrm{~V}$ in contact and tapping mode experiments. Figure 5 shows the height of $\mathrm{Si}$ oxide dots as a function of oxidation time. As the rate constants are assumed to be $k_{1}$ $=4.3 \times 10^{0} \mathrm{~s}^{-1}, k_{3}=3.5 \times 10^{-3} \mathrm{~s}^{-1}$, and $k_{4}=2.5 \times 10^{-1} \mathrm{~s}^{-1}$, the model with $\gamma=0.4$ provides a good fit to the experimental data of contact mode oxidation. On the other hand, in tapping mode experiments, the experimental data are clearly fitted by the model assuming that the rate constants are $k_{1}$ $=1.3 \times 10^{1} \mathrm{~s}^{-1}, k_{3}=4.2 \times 10^{-2} \mathrm{~s}^{-1}$, and $k_{4}=1.3 \times 10^{0} \mathrm{~s}^{-1}$.

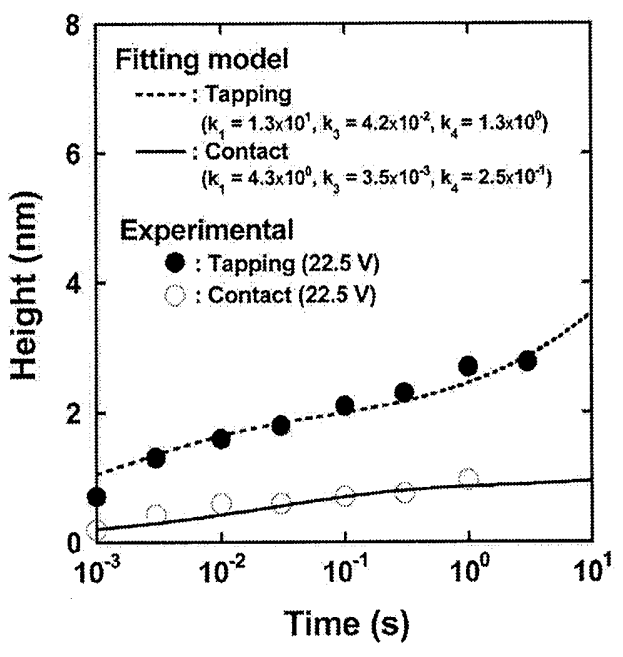

FIG. 5. Height of Si oxide dots formed by contact and tapping mode SPM local oxidation as a function of oxidation time. Solid and open circles show the experimental data of tapping and contact mode SPM local oxidation, respectively.

This result indicates that all the rate constants in tapping mode oxidation are larger than those of contact mode oxidation, suggesting that the anodic oxidation is enhanced in tapping mode operation. In contact mode oxidation, it is considered that the space charge is accumulated in the oxide and prevents further growth of the oxide. ${ }^{20}$ The cantilever vibrates at the resonance frequency in tapping mode operation, so that the electric field strength between SPM tip and sample surface is strongly modulated in tapping mode oxidation with dc bias voltage. In other words, the electric field depends on the distance between the SPM tip and sample surface and is stronger when the tip is closer to the surface. It is known that the effective diffusivity of $\mathrm{H}^{+}$in $p$-type silicon increases with the increase of electric field. ${ }^{21}$ If the similar electric field effect occurs during the $\mathrm{Si}$ oxide formation in tapping mode operation, the diffusivity of space charges in the oxide may be enhanced with the oscillating motion of the cantilever. Therefore, it seems that space charges involved within the $\mathrm{Si}$ oxide are easily neutralized and released by the electric field effect. This effect could play an important role for stable SPM oxidation.

\section{CONCLUSIONS}

We investigated the influence of SPM operation modes on Si oxide formation between contact and tapping mode SPM local oxidation experiments. Under the same oxidation conditions with the same SPM tip, the average FWHM and the STD of the Si oxide wire fabricated by tapping mode oxidation are 11.0 and $2.1 \mathrm{~nm}$, respectively, which are smaller than those of contact mode oxidation. Furthermore, we exhibited the model of tapping mode SPM local oxidation based on the oxidation ratio and the rate constant. From the model, the oxidation ratio is estimated to be $10 \%-16 \%$ in tapping mode oxidation with $10 \mathrm{~nm}$ resolution. The rate constants of tap- 
ping mode oxidation are larger than those of contact mode oxidation, which suggests the formation of small and uniform oxide in tapping mode operation. These results imply that tapping mode SPM local oxidation is a suitable technique for the fabrication of nanometer-scale $\mathrm{Si}$ oxide wires with higher controllability and better size uniformity.

\section{ACKNOWLEDGMENT}

This work was carried out under the support program for improving graduate school education of "Human Resource Development Program for Scientific Powerhouse," in Tokyo University of Agriculture and Technology.

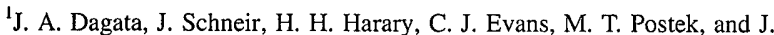
Bennett, Appl. Phys. Lett. 56, 2001 (1990).

${ }^{2}$ J. Shirakashi, K. Matsumoto, N. Miura, and M. Konagai, Jpn. J. Appl. Phys., Part 1 37, 1594 (1998).

${ }^{3}$ J. Shirakashi, K. Matsumoto, N. Miura, and M. Konagai, Appl. Phys. Lett. 72, 1893 (1998).

${ }^{4}$ Y. Tomoda, Y. Shibata, J. Shirakashi, and Y. Takemura, J. Appl. Phys. 99, $08 \mathrm{~T} 312(2006)$

${ }^{5}$ Y. Tomoda, S. Kayashima, T. Ogino, M. Motoyama, Y. Takemura, and J. Shirakashi, J. Magn. Magn. Mater. 310, e641 (2007).

${ }^{6}$ R. Garcia, M. Calleja, and H. Rohrer, J. Appl. Phys. 86, 1898 (1999).
${ }^{7}$ H. Jungblut, D. Wille, and H. J. Lewerenz, Appl. Phys. Lett. 78, 168 (2001).

${ }^{8}$ T. Hattori, Y. Ejiri, K. Saito, and M. Yasutake, J. Vac. Sci. Technol. A 12 2586 (1994)

${ }^{9}$ M. Tello and R. Garcia, Appl. Phys. Lett. 79, 424 (2001).

${ }^{10}$ J. A. Dagata, T. Inoue, J. Itou, and H. Yokoyama, Appl. Phys. Lett. 73, 271 (1998).

${ }^{11}$ Y. Shibata, Y. Tomoda, J. Shirakashi, and Y. Takemura, Abstract of the 50th Conference on Magnetism and Magnetic Materials, 2005 (unpublished), p. 214

${ }^{12}$ S. Nishimura, Y. Takemura, and J. Shirakashi, J. Phys.: Conf. Ser. 61, 1066 (2007).

${ }^{13}$ S. Nishimura, T. Ogino, Y. Takemura, and J. Shirakashi, J. Phys.: Conf. Ser. 100, 052021 (2008)

${ }^{14} \mathrm{Ph}$. Avouris, T. Hertel, and R. Martel, Appl. Phys. Lett. 71, 285 (1997).

${ }^{15}$ D. Stievenard, P. A. Fontaine, and E. Dubois, Appl. Phys. Lett. 70, 3272 (1997).

${ }^{16}$ J. A. Dagata, F. Perez-Murano, G. Abadal, K. Morimoto, T. Inoue, J. Itoh, and H. Yokoyama, Appl. Phys. Lett. 76, 2710 (2000).

${ }^{17} \mathrm{~S}$. Nishimura, T. Ogino, Y. Takemura, and J. Shirakashi, Jpn. J. Appl. Phys., Part 1 47, 718 (2008)

${ }^{18} \mathrm{Ph}$. Avouris, R. Martel, T. Hertel, and R. Sandstrom, Appl. Phys. A: Mater. Sci. Process. 66, S659 (1998).

${ }^{19}$ R. A. Alberty and W. G. Miller, J. Chem. Phys. 26, 1231 (1957).

${ }^{20} \mathrm{~J}$. A. Dagata, T. Inoue, J. Itoh, K. Matsumoto, and H. Yokoyama, J. Appl. Phys. 84, 6891 (1998).

${ }^{2 \mathrm{I}}$ Y. L. Huang, B. Wdowiak, R. Job, Y. Ma, and W. R. Fahrner, J. Electrochem. Soc. 151, G564 (2004). 